\title{
The Yeast Genome Directory
}

This directory was made possible by a unique international collaboration between the 633 scientists whose names appear below. It represents both the first published description of the complete sequence of most chromsomes from Saccharomyces cerevisiae, and the first published overview of the entire sequence. As such, the authors would like future papers referring to the entire sequence and/or its contents to cite this directory; future papers referring to the sequence of individual chromosomes should refer to the papers listed at the head of page 9. The authors' affiliations appear in the papers describing the individual chromosomes.

A. Goffeau, R. Aert, M. L. Agostini-Carbone, A. Ahmed, M. Aigle, L. Alberghina, K. Albermann, M. Albers, M. Aldea, D. Alexandraki, G. Aljinovic, E. Allen, J. Alt-Mörbe, B. André, S. Andrews, W. Ansorge, G. Antoine, R. Anwar, A. Aparicio, R. Araujo, J. Arino, F. Arnold, J. Arroyo, E. Aviles, U. Backes, M. C. Baclet, K. Badcock, A. Bahr, V. Baladron, J. P. G. Ballesta, A. T. Bankier, A. Banrevi, M. Bargues, L. Baron, T. Barreiros, B. G. Barrell, C. Barthe, A. B. Barton, A. Baur, A.-M. Bécam, A. Becker, I. Becker, J. Beinhauer, V. Benes, P. Benit, G. Berben, E. Bergantino, P. Bergez, A. Berno, I. Bertani, N. Biteau, A. J. Bjourson, H. Blöcker, C. Blugeon, C. Bohn, E. Boles, P. A. Bolle, M. Bolotin-Fukuhara, R. Bordonné, J. Boskovic, P. Bossier, D. Botstein, G. Bou, S. Bowman, J. Boyer, P. Brandt, T. Brandt, M. Brendel, T. Brennan, R. Brinkman, A. Brown, A. J. P. Brown, D. Brown, M. Brückner, C. V. Bruschi, J. M. Buhler, M. J. Buitrago, F. Bussereau, H. Bussey, A. Camasses, C. Carcano, G. Carignani, J. Carpenter, A. Casamayor, C. Casas, L. Castagnoli, H. Cederberg, E. Cerdan, N. Chalwatzis, R. Chanet, E. Chen, G. Chéret, J. M. Cherry,

T. Chillingworth, C. Christiansen, J.-C. Chuat, E. Chung, C. Churcher, C. M. Churcher, M. W. Clark, M. L. Clemente, A. Coblenz,

M. Coglievina, E. Coissac, L. Colleaux, R. Connor, R. Contreras, J. Cooper, T. Copsey, F. Coster, R. Coster, J. Couch, M. Crouzet, C. Cziepluch, B. Daignan-Fornier, F. Dal Paro, D. V. Dang,

M. D’Angelo, C. J. Davies, K. Davis, R. W. Davis, A. De Antoni, S. Dear, K. Dedman, E. Defoor, M. De Haan, Th. Delaveau, S. Del Bino, M. Delgado, H. Delius, D. Delneri, F. Del Rey, J. Demolder, N. Démolis, K. Devlin, P. de Wergifosse, F. S. Dietrich, H. Ding, C. Dion, T. Dipaolo, F. Doignon, C. Doira, H. Domdey, J. Dover, Z. Du, E. Dubois, B. Dujon, M. Duncan, P. Durand, A. Düsterhöft, S. Düsterhus, T. Eki,

M. El Bakkoury, L. G. Eide, K.-D. Entian, P. Eraso, D. Erdmann, H. Erfle, V.Escribano, M. Esteban, L. Fabiani, F. Fabre, C. Fairhead, B. Fartmann, A. Favello, G. Faye, H. Feldmann, L. Fernandes, F. Feroli,

M. Feuermann, T. Fiedler, W. Fiers, U. N. Fleig, M. Flöth, G. M. Fobo, N. Fortin, F. Foury, M. C. Francingues-Gaillard, L. Franco, A. Fraser, J.D. Friesen, C. Fritz, L. Frontali, H. Fukuhara, L. Fulton, L. J. Fuller, C. Gabel, C. Gaillardin, L. Gaillon, F. Galibert, F. Galisson, P. Galland, F.-J. Gamo, C. Gancedo, J. M. Garcia-Cantalejo, M. I. García-Gonzalez, J. J. Garcia-Ramirez, M. García-Saéz, H. Gassenhuber, M. Gatius, S. Gattung, C. Geisel, M. E. Gent, S. Gentles, M. Ghazvini, D. Gigot, V. Gilliquet, N. Glansdorff, A. Gómez-Peris, A. Gonzaléz, S. E. Goulding, C. Granotier, T. Greco, M. Grenson, P. Grisanti, L. A. Grivell,

D. Grothues, U. Gueldener, P. Guerreiro, E. Guzman, M. Haasemann, B. Habbig, H. Hagiwara, J. Hall, K. Hallsworth, N. Hamlin, N. J. Hand, V. Hanemann, J. Hani, T. Hankeln, M. Hansen, D. Harris, D. E. Harris, G. Hartzell, D. Hatat, U. Hattenhorst, J. Hawkins, U. Hebling, J. Hegemann, C. Hein, A. Hennemann, K. Hennessy, C. J. Herbert, K. Hernandez, Y. Hernando, E. Herrero, K. Heumann, D. Heuss- Neitzel, N. Hewitt, R. Hiesel, H. Hilbert, F. Hilger, L. Hillier, C. Ho, J. Hoenicka, B. Hofmann, J. Hoheisel, S. Hohmann, C. P. Hollenberg, K. Holmstrøm, O. Horaitis, T. S. Horsnell, M.-E. Huang, B. Hughes, S. Hunicke-Smith, S. Hunt, S. E. Hunt, K. Huse, R. W. Hyman, F. Iborra, K. J. Indge, I. Iraqui Houssaini, K. Isono, C. Jacq, M. Jacquet, A. Jacquier, K. Jagels, W. Jäger, C. M. James, J. C. Jauniaux, Y. Jia, M. Jier, A. Jimenez, D. Johnson, L. Johnston, M. Johnston, M. Jones, J.-L. Jonniaux, D. B. Kaback, T. Kallesøe, S. Kalman, A. Kalogeropoulos, L. KarpfingerHartl, D. Kashkari, C. Katsoulou, A. Kayser, A. Kelly, T. Keng, H. Keuchel, P. Kiesau, L. Kirchrath, J. Kirsten, K. Kleine, U. Kleinhans, R. Klima, C. Komp, E. Kordes, S. Korol, P. Kötter, C. Krämer, B. Kramer, P. Kreisl, T. Kucaba, H. Kuester, O. Kurdi, P. Laamanen, M. J. Lafuente,
O. Landt, G. Lanfranchi, Y. Langston, D. Lashkari, P. Latreille, G. Lauquin, T. Le, P. Legrain, Y. Legros, A. Lepingle, H. Lesveque, H. Leuther, H. Lew, C. Lewis, Z. Y. Li, S. Liebl, A. Lin, D. Lin, M. Logghe, A. J. E. Lohan, E. J. Louis, G. Lucchini, K. Lutzenkirchen, R. Lyck, G. Lye, A. C. Maarse, M. J. Maat, C. Macri, A. Madania, M. Maftahi, A. Maia e Silva, E. Maillier, L. Mallet, G. Mannhaupt, V. Manus, R. Marathe, C. Marck, A. Marconi, E. Mardis, E. Martegani, R. Martin, A. Mathieu, C. T. C. Maurer, M. J. Mazón, C. Mazzoni, D. McConnell, S. McDonald, R. A. McKee, A. D. K. McReynolds, P. Melchioretto, S. Menezes, F. Messenguy, H. W. Mewes, G. Michaux, N. Miller, O. Minenkova, T. Miosga, S. Mirtipati, S. Möller-Rieker, D. Möstl, F. Molemans, A. Monnet, A-L. Monnier, M. A. Montague, M. Moro, D. Mosedale, D. Möstl, S. Moule, L. Mouser, Y. Murakami, S. Müller-Auer, J. Mulligan, L. Murphy, M. Muzi Falconi, M. Naitou, K. Nakahara, A. Namath, F. Nasr, L. Navas, A. Nawrocki, J. Nelson, U. Nentwich, P. Netter, R. Neu, C. S. Newlon, M. Nhan, J.-M. Nicaud, R. K. Niedenthal, C. Nombela, D. Noone, R. Norgren, B. Nußbaumer, B. Obermaier, C. Odell, P. Öfner, C. Oh, K. Oliver, S. G. Oliver, B. F. Ouellette, M. Ozawa, V. Paces, C. Pallier, D. Pandolfo, L. Panzeri, S. Paoluzi, A. G. Parle-Mcdermott, S. Pascolo, N. Patricio, A. Pauley, L. Paulin, B. M. Pearson, D. Pearson, D. Peluso, J. Perea, M. Pérez-Alonso, J. E. Pérez-Ortin, A. Perrin, F. X. Petel, B. Pettersson, F. Pfeiffer, P. Philippsen, A. Piérard, E. Piravandi, R. J. Planta, P. Plevani, O. Poch, B. Poetsch, F. M. Pohl, T. M. Pohl, R. Pöhlmann, R. Poirey, D. Portetelle, F. Portillo, S. Potier, M. Proft, H. Prydz, A. Pujol, B. Purnelle, V. Puzos, M. A. Rajandream, M. Ramezani Rad, S. W. Rasmussen, A. Raynal, S. Rechmann, M. Remacha, J. L. Revuelta, P. Rice, G-F. Richard, P. Richterich, M. Rieger, L. Rifken, L. Riles, T. Rinaldi, M. Rinke, A. B. Roberts, D. Roberts, F. Rodriguez, E. Rodriguez-Belmonte, C. RodriguezPousada, A. M. Rodriguez-Torres, M. Rose, R. Rossau, N. Rowley, T. Rupp, M. Ruzzi, W. Saeger, J. E. Saiz, M. Saliola, D. Salom, H. P. Saluz, M. SánchezPerez, M. A. Santos, E. Sanz, J. E. Sanz, A.-M. Saren, F. Sartorello, M. Sasanuma, S-I. Sasanuma, T. Scarcez, I. Schaaf-Gerstenschläger, B. Schäfer, M. Schäfer, M. Scharfe, B. Scherens, N. Schroff, M. Sen-Gupta, T. Shibata, T. Schmidheini, E. R. Schmidt, C. Schneider, P. Scholler, S. Schramm, A. Schreer, M. Schröder, C. Schwager, S. Schwarz, C. Schwarzlose, B. Schweitzer, M. Schweizer, A-M. Sdicu, P. Sehl, C. Sensen, J. G. Sgouros, T. Shogren, L. Shore, Y. Shu, J. Skala, J. Skelton, P. P. Slonimski, P. H. M. Smit, V. Smith, H. Soares, E. Soeda, A. Soler-Mira, F. Sor, N. Soriano, J. L. Souciet, C. Soustelle,

R. Spiegelberg, L. I. Stateva, H. Y. Steensma, J. Stegemann, S. Steiner, L. Stellyes, F. Sterky, R. K. Storms, H. St. Peter, R. Stucka, A. Taich, E. Talla, I. Tarassov, H. Tashiro, P. Taylor, C. Teodoru, H. Tettelin, A. Thierry, G. Thireos, E. Tobiasch, D. Tovan, E. Trevaskis, Y. Tsuchiya, M. Tzermia, M. Uhlen, A. Underwood, M. Unseld, J. H. M. Urbanus, A. Urrestarazu, S. Ushinsky, M. Valens, G. Valle, A. Van Broekhoven, M. Vandenbol, Q. J. M. Van Der Aart, C. G. Van Der Linden, L. Van Dyck, M. Vanoni, J. C. Van Vliet-Reedijk, A. Vassarotti, M. Vaudin, K. Vaughan, P. Verhasselt, I. Vetter, F. Vierendeels, D. Vignati, C. Vilela, S. Vissers, C. Vleck, D. T. Vo, D. H. Vo, M. Voet, G. Volckaert, D. Von Wettstein, H. Voss, P. Vreken, G. Wagner, S. V. Walsh, R. Wambutt, H. Wang, Y. Wang, J. R. Warmington, R. Waterston, M. D. Watson, N. Weber, E. Wedler, H. Wedler, Y. Wei, S. Whitehead, B. L. Wicksteed, S. Wiemann, L. Wilcox, C. Wilson, R. Wilson, A. Winant, E. Winnett, B. Winsor, P. Wipfli, S. Wölfl, P. Wohldman, K. Wolf, K. H. Wolfe, L. F. Wright, H. Wurst, G. Xu, M. Yamasaki, M. A. Yelton, K. Yokohama, A. Yoshikawa, S. Yuping, P. Zaccaria, M. Zagulski, F. K. Zimmermann, J.Zimmermann, M.Zimmermann, W-W. Zhong, A. Zollner, E. Zumstein. 